\title{
First elemental imaging experiments on a combined PGAI and NT setup at the Budapest Research Reactor
}

\author{
T. Belgya, ${ }^{1 *}$ Z. Kis, ${ }^{1}$ L. Szentmiklósi, ${ }^{1}$ Zs. Kasztovszky, ${ }^{1}$ P. Kudejova, ${ }^{2}$ R. Schulze, ${ }^{2}$ T. Materna, ${ }^{3}$ \\ G. Festa, ${ }^{4}$ P. A. Caroppi, ${ }^{4}$ the Ancient Charm Collaboration \\ ${ }^{I}$ Institute of Isotopes HAS, Budapest, P.O. Box 77, H-1525 Budapest, Hungary \\ ${ }^{2}$ University of Cologne, Germany \\ ${ }^{3}$ Institut Laue-Langevin, France \\ ${ }^{4}$ University of Tor Vergata, Rome, Italy
}

(Received July 10, 2008)

The European collaboration "ANCIENT CHARM" (http://ancient-charm.neutron-eu.net/ach/) aims to develop new non-destructive neutron techniques to image the internal composition of complex archaeological objects in order to answer various archaeological questions. Among these techniques, prompt gamma activation imaging (PGAI) and neutron tomography (NT) form a unique combination which can determine the 3D distribution of most elements in objects with a non-destructive procedure. A spatial resolution better than $2 \mathrm{~mm}$ has already been achieved in a moderately scattering matrix material.

\section{Introduction}

Noninvasive or nondestructive imaging of the interior of macroscopic objects goes back to the famous discovery of Wilhelm Conrad RönTGEN. ${ }^{1}$ X-rays penetrating the macroscopic object interact with the electrons of its constituent atoms and scatter out from their initial direction or disappear by absorption. The remaining X-rays then interact with the detector behind the object. The most frequently used detector is a Röntgen film, which records the transmitted X-rays by a photochemical reaction. The blacker film areas correspond to regions of lower electron density in the object. Thus X-rays measure the projected electron density between the source and the detector.

Later, more sophisticated methodologies were worked out for X-ray imaging to obtain 3D images of a macroscopic object. The most advanced technology is called computed tomography (CT), which yields the electron density not as a projection but for small volumes of the body (the so called voxels), and this is widely used in medical examinations. It was invented by HounsFIELD, ${ }^{2}$ who was awarded a Nobel Prize in Medicine. In addition to the X-ray tomography, there are now several other techniques which can make a 3D image of the interior of objects, such as electron spin resonance (ESR), nuclear magnetic resonance (NMR), positron emission tomography (PET), etc. These widely used medical imaging techniques are able to provide an image of some selected materials in the bodies, but none of the above techniques are able to readily provide chemical element information about the object.

Prompt gamma neutron activation analysis (PGAA or PGNAA) is a nondestructive elemental analysis method. ${ }^{3}$ One of its advantages among the numerous nondestructive elemental analysis methods is that both the neutrons and the observed gamma-rays can penetrate deeply in the materials and thus are able to provide information from several centimeter depths of the objects. Owing to this feature, PGAA provides the bulk composition of an object. If we modify the original idea of the PGAA, i.e., irradiating the object with a narrow beam of neutrons and observing it with a large detector, we can obtain spatial elemental information about the object. The idea is not completely new. In 1987, SPYROU, KUSMINARTO and NICOLAOU ${ }^{4}$ have already published a $2 \mathrm{D}$ reconstruction of an object using narrow neutron beam and large detector.

The idea was considerably extended for studying archaeological objects when the European Union supported project, "ANCIENT CHARM" (AC), was launched. 5 The goal of the project is to study the elemental (or even isotopic) and internal tension distribution in archaeological objects in 3D using slowand epithermal-neutron based imaging techniques. The archaeological objects were chosen for the topic of AC project, because they are our primary interest and require non-destructive methods. At the level of our present knowledge and capability, the methods are not yet applicable to living organisms. An overview description of the full project is given by GORINI, ${ }^{6}$ the consortium leader of the project.

Many different kinds of experiments have already been performed in the project. $^{7-10}$ The PGAA-NIPS facilities of the Budapest Research Reactor is intended to provide the first elemental image in the project and for this reason we have performed a large number of experiments to achieve this goal. All of the above experiments have focused up to now on the development of the $3 \mathrm{D}$ imaging on benchmark samples created for the

\footnotetext{
*E-mail: belgya@iki.kfki.hu
} 
project by archaeologists. The benchmark objects have compositions unknown to the experimentalists and simple geometrical shapes in order to find out the weaknesses and strengths of the new methods.

This paper will concentrate on a new quasi 3D imaging technique achieved by PGAA at the NIPS facility; we call this prompt gamma activation imaging (PGAI). The idea of PGAI has already been published. ${ }^{9}$ However its realization has required a great deal of hardware and software development. First a simple object with known composition was selected for study in order to be able to understand the characteristics of this 3D imaging technique. The results of this study are reported here.

\section{The experimental setup}

The detailed description and the basic characteristics of the experimental setup is presented in a separate paper to this conference. ${ }^{11}$ Here we give only a short summary for clarity. The neutron source in our case is the 10-MW Budapest Research Reactor. The neutrons are cooled in a cold source and guided to the experimental area with a supermirror guide. Just before neutrons reach the experimental area they are divided into two beams by collimators. The upper one serves the PGAA facility, while the lower beam goes further and is used in the NIPS facility. The purpose of the NIPS facility is to accommodate various setups; consequently it was used as a site for the PGAI/NT equipment. A recent detailed description for these facilities is given by RÉVAY et al. ${ }^{3}$

The PGAI/NT setup consists of a neutron collimator with fixed horizontal width of $2 \mathrm{~mm}$ and a variable vertical opening. The target chamber was made from ${ }^{6} \mathrm{Li}$ enriched polymer with $20 \times 20 \times 20 \mathrm{~cm}^{3}$ size. Its entrance window was close to the neutron collimator and the exit window was closely followed by the neutron tomograph (NT) device. Behind the NT a beam stop was constructed with sufficient shielding. Perpendicular to the neutron beam a $15 \mathrm{~cm}$ thick, variable aperture gamma-ray-collimator was placed next to the wall of the target chamber and in front of a high purity germanium gamma-ray detector. The neutron- and gammacollimator sizes were equally set to $20 \times 2 \mathrm{~mm}^{2}$ (vertical $\times$ horizontal) sizes in the experiments presented here. The sample was positioned relative to the isocenter (the middle point of the cross section of the neutron beam and the gamma collimator solid angle view) using an $X Y Z \omega$ moving table. The volume of the above inter section is called the isovolume, and is the source of the observed gamma-rays arising from neutron capture. The whole system (moving table, NT and batch gamma acquisition) was controlled with a single computer program designed and developed locally and embedded in the gamma acquisition software provided by the manufacturer for the hardware.

\section{Experiments on a simple sample}

During the design phase of the setup we have already simulated it with Monte Carlo calculations. It was predicted that a $22 \times 2 \mathrm{~mm}^{3}$ isovolume would have provided very low gamma count rate for the most common materials of archaeological samples with our present neutron flux at the NIPS facility. For this reason we used vertically elongated neutron and gamma-ray collimators, which increased the rate by a factor of 100 . This arrangement could be used to study the elemental distribution only for vertically translational symmetric objects. For this reason, we created a horizontally inhomogeneous sample with this symmetry. It was an aluminum cylinder with $12 \mathrm{~mm}$ diameter and $40 \mathrm{~mm}$ length. Parallel with its axis we drilled two holes of $2 \mathrm{~mm}$ diameter and with centers $2 \mathrm{~mm}$ from the aluminum symmetry axis. The holes' centers and the symmetry axis of the aluminum cylinder were on a single line. In one of the holes a copper wire of $1.6 \mathrm{~mm}$ diameter was placed while in the other hole an iron nail of $1.9 \mathrm{~mm}$ diameter was inserted, as sketched in Fig. 1.

The symmetry axis of the sample was approximately placed in the isocenter of the experimental system on the top of the moving table. Scanning of this elongated sample was performed on a planar grid being perpendicular to the axis of the cylinder. The grid points extended from $-8 \mathrm{~mm}$ to $8 \mathrm{~mm}$ in both $\mathrm{X}$ and $\mathrm{Y}$ horizontal directions. The $\mathrm{X}$-axis is parallel to the neutron beam and the $\mathrm{Z}$-axis is parallel to the directions of the detected gamma-rays. Steps of $2 \mathrm{~mm}$ were chosen for the two outer grid settings (to obtain background counts) while $1 \mathrm{~mm}$ was used in the inner $-4 \mathrm{~mm}$ to $4 \mathrm{~mm}$ region.

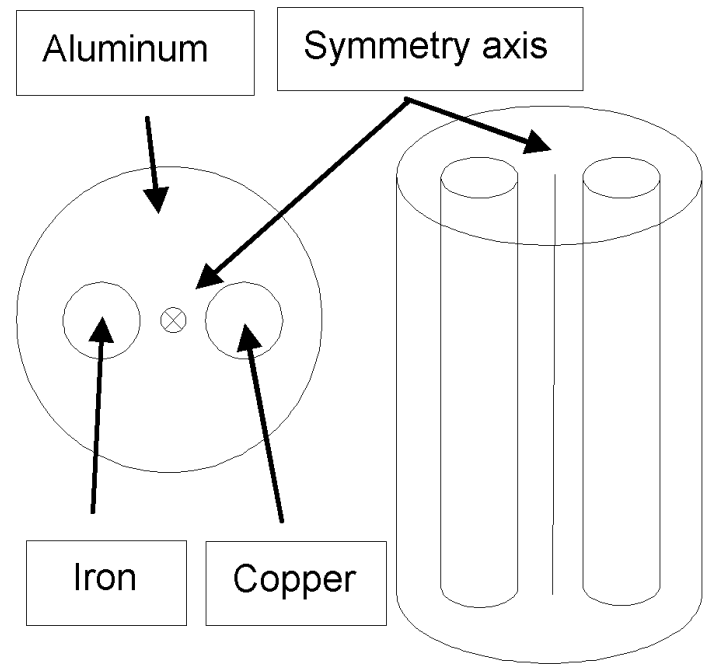

Fig. 1. The elongated aluminum sample with two rods 
The inner region covers the interesting volume of the sample. All together we measured at 137 grid points using the batch processing acquisition mode of the experimental system. At each grid point we acquired a gamma-ray spectrum for 2500 seconds. The whole run lasted for 4 days. From this time span it can be seen that a complete 3D scanning of a more complex object would last for an unacceptably long time with the present system.

\section{Results and discussion}

This large amount of data was analyzed with the batch mode of HyperLab software ${ }^{12}$ to obtain peak areas for the 3 elements contained in the sample. The peak areas of the most intense peaks of these elements were than correlated with the grid point coordinates to produce a $2 \mathrm{D}$ (quasi 3D) elemental distribution of the target. These peaks were the $1778-\mathrm{keV}$ decay line of aluminum, and the $352-\mathrm{keV}$ and $278-\mathrm{keV}$ prompt lines of $\mathrm{Fe}$ and $\mathrm{Cu}$, respectively. Before production of the peak area maps we calculated peak areas at the missing equidistant grid-points, by averaging the areas of the neighboring grid points in the $2 \mathrm{~mm}$ step region, to generate a common $1 \mathrm{~mm}$ grid for the whole $16 \mathrm{~mm}$ by $16 \mathrm{~mm}$ area. The plot of this 17 by 17 matrix for each element is shown in Fig. 2.

Several characteristics of this 3D imaging technique can be learned from Fig. 2:

(a) In the aluminum cylinder at the places of the $\mathrm{Fe}$ and $\mathrm{Cu}$ rods there seems to be some intensity deficit as it should be, more at the more absorbing $\mathrm{Cu}$ rod and less at the Fe rod. This picture may be obscured by the fact that the $\mathrm{Al}$ decay line intensity does not go to zero when the neutron beam is changed to the next position.

(b) As can be seen from the panels, the images of the cylindrically symmetric objects are elongated in the X direction (the direction of the neutron beam), which is due to the gamma-collimator transmission. ${ }^{11}$ FWHM being larger than the n-beam FWHM.

(c) The aluminum intensity does not disappear when the neutron beam does not hit the cylinder. This can be explained by the constant background caused by the neighboring aluminum construction materials.

(d) There seems to be some misalignment of the aluminum cylinder relative to the isocenter. To improve the quality of the images, corrections for the neutron distribution, gamma transmission through the collimator, scattering and self-absorption for both neutrons and gamma-rays should be applied. The development of the methodology of these calculations is in progress.

(e) The intensity plot of the Fe-rod (middle panel) seems to be wider than the Cu-rod (lower panel) as it should be from the geometrical sizes of the rods. This means that it is possible to determine positions and sizes with a precision smaller than the collimator widths when the grid distances are smaller than that. A Gaussian peak fit to the experimental data at the $X=0$ cut gives the following results presented in Table 1. The nominal geometrical ratio of the $\mathrm{Fe} / \mathrm{Cu}$ diameter is $1.9 / 1.6=1.19$ while the fitted one is 1.14 . The nominal separation of the two rods is $4 \mathrm{~mm}$, while the fitted value is $2.38+1.77=4.15 \mathrm{~mm}$. They are in good agreement taking into account all of the possible sources of uncertainties.
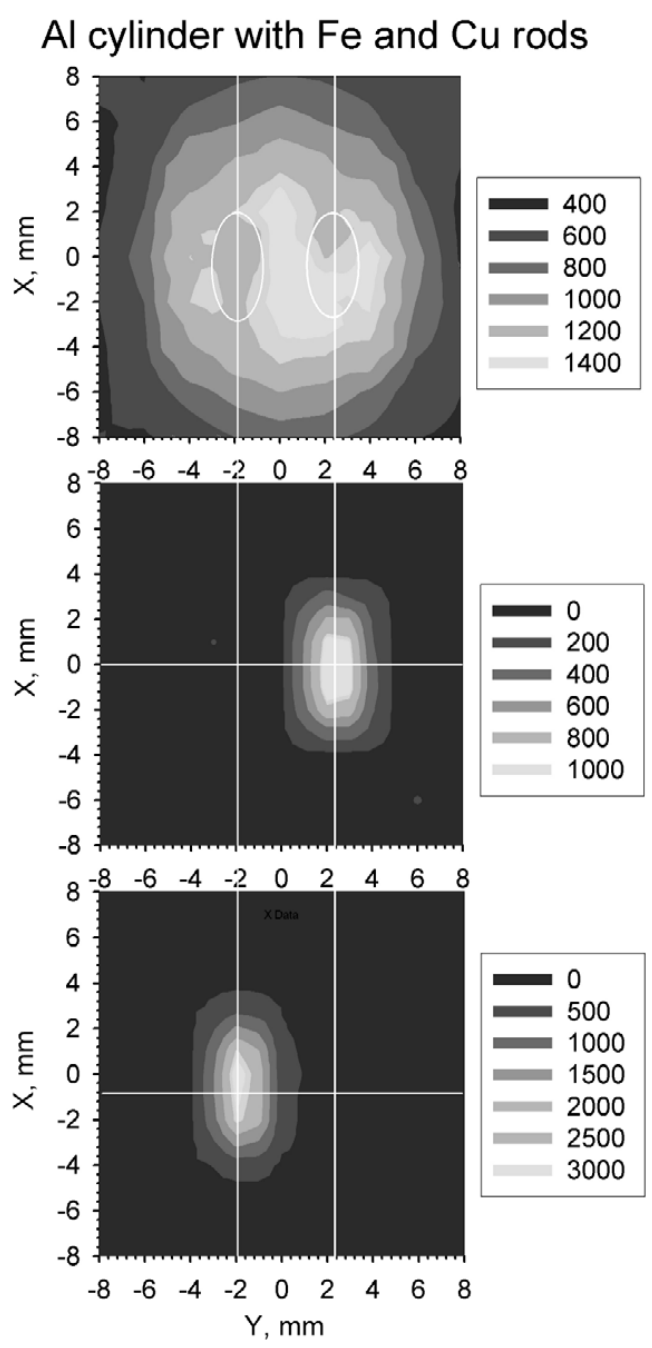

Fig. 2. Top panel is the peak area map of the aluminum cylinder. The middle panel is for the $\mathrm{Fe}$, while the lower panel is for the $\mathrm{Cu}$ rods. The vertical and horizontal lines help to project the middle of the elemental distributions to the axis and on each other

Table 1. Parameters of the Gaussian peak fit to the experimental intensity data at the $\mathrm{X}=0$ cut to the data presented in Fig. 2

\begin{tabular}{lcccc}
\hline \multirow{2}{*}{ Parameter } & \multicolumn{2}{c}{$\mathrm{Fe}$} & \multicolumn{2}{c}{$\mathrm{Cu}$} \\
\cline { 2 - 5 } & Value & Std. & Value & Std \\
\hline Amplitude & 1226 & 26 & 3185 & 53 \\
FWHM (mm) & 2.34 & 0.06 & 2.06 & 0.04 \\
Position (mm) & 2.38 & 0.02 & -1.77 & 0.02 \\
\hline
\end{tabular}



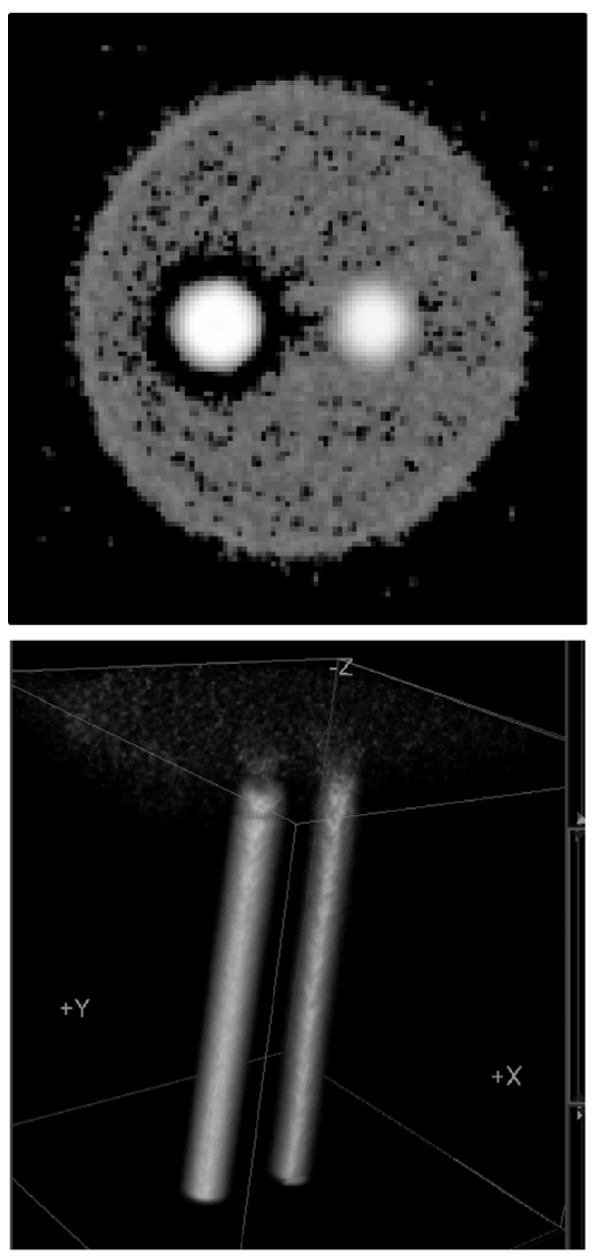

Fig. 3. A neutron tomograph slice across the vertical symmetry axis of the $\mathrm{Al}$ cylinder sample and a $3 \mathrm{D}$ view of the iron and copper rods

Based on the NT radiographs taken at many different angles a $3 \mathrm{D}$ image of the object was reconstructed using the filtered back-projection technique (or other algorithm). This kind of reconstruction is equivalent to that applied in medical imaging and it yields the neutron absorption in the voxels. Using the spatial coordinates and the so called grey value of each voxel we can generate arbitrary slices of various thicknesses and produce volumetric images of the original object. The resulting images were examined with VGStudio Max 1.2 to highlight the interesting parts of the sample. A cut perpendicular to the symmetry axis and a 3D picture of the iron and copper rods are shown in Fig. 3.

Identifying the interesting sections can help to position them in the isovolume, which greatly shortens the time needed to collect comprehensive elemental information about the object. Our aim is to speed up the measurements in this way on unknown-composition objects, by using the NT coordinates as driving input for the $\mathrm{XYZ} \omega$ moving table. A manual version of this idea has been experimentally performed; however the presentation of these results is out of the scope of this paper.

\section{Conclusions}

A working PGAI-NT facility was built, with the aim of scrutinizing the internal structure and elemental distribution of archeological objects. Methods were worked out to acquire and process a large number of gamma-spectra. Quasi 3D elemental compositions of a known benchmark object were measured in a grid scan. The raw elemental map qualitatively agrees well with the known composition of the object, and quantitatively reproduces the geometrical distribution of the different elements. This shows that the idea of 3D elementalmapping with PGAI is working well. Reduction of the background should improve the signal to background ratio.

Efforts to combine the complementary features of neutron tomography and PGAI requires more work and could lead to an efficient method for investigating valuable cultural heritage objects.

Thanks for the support of EU FP 6 ANCIENT CHARM Project, Contract No. 15311 and the NAP VENEUS05 Project, Contract No. OMFB-00648/2005.

\section{References}

1. W. C. RÖNTGEN, Nature, 53 (1896) 274.

2. G. N. HounsFIELD, British J. Radiol., 46 (1973) 1016.

3. Z. Révay, T. Belgya, Z. Kasztovszky, J. L. Weil, G. L. Molnár, Nucl. Instr. Meth. Phys. Res., B213 (2004) 385.

4. N. M. Spyrou, Kusminarto, G. E. Nicolaou, J. Radioanal. Nucl. Chem., 112 (1987) 57.

5. Analysis by Neutron Resonant Capture Imaging and Other Emerging Neutron Techniques: New Cultural Heritage and Archaeological Research Methods, 2005, available: http://ancientcharm.neutron-eu.net/ach

6. G. GORINI and The Ancient Charm Collaboration, Nuovo Cimento, (2007) 1.

7. P. Kudejova, J. Ciyek, R. Schulze, J. Jolie, B. Schillinger, K. Lorenz, M. Mühlbauer, B. Masschaele, M. Dierick, J. VlassenBroecK, Notizario Neutroni E Luce Di Sincrotrone, 12 (2007) 6

8. G. Festa, C. Andreani, A. Filabozzi, D. Malfitana, J. PoBlome, Archeometriai Mühely, 2 (2006) 32.

9. Z. KasztovszKy, T. Belgya, Archeometriai Műhely, 1 (2006) 12.

10. W. Kockelmann, A. Kirfel, Archeometriai Mühely, 2 (2006) 1.

11. T. Belgya, Z. KIS, L. Szentmiklósi, Z. Kasztovszky, G. Festa, L. Andreanelli, M. P. DE Pascale, A. Pietropaolo, P. Kudejova, R. Schulze, T. Materna, and The Ancient Charm Collaboration, J. Radioanal. Nucl. Chem., 278 (2008) 713.

12. A. Simonits, J. ÖstöR, S. KÁlvin, B. FAzeKas, J. Radional. Nucl. Chem., 257 (2003) 589, http://www.hlabsoft.com 